\title{
MAC-Aware and Power-Aware Image Aggregation Scheme in Wireless Visual Sensor Networks
}

\author{
Yui-Liang Chen and Hong-Hsu Yen \\ Department of Information Management, Shih Hsin University, No. 1, Lane 17, Section 1, Mu-Cha Road, Taipei City 116, Taiwan
}

Correspondence should be addressed to Yui-Liang Chen; ychen@cc.shu.edu.tw

Received 19 July 2012; Revised 10 January 2013; Accepted 15 January 2013

Academic Editor: Christos Riziotis

Copyright (C) 2013 Y.-L. Chen and H.-H. Yen. This is an open access article distributed under the Creative Commons Attribution License, which permits unrestricted use, distribution, and reproduction in any medium, provided the original work is properly cited.

\begin{abstract}
Traditional wireless sensor networks (WSNs) transmit the scalar data (e.g., temperature and irradiation) to the sink node. A new wireless visual sensor network (WVSN) that can transmit images data is a more promising solution than the WSN on sensing, detecting, and monitoring the environment to enhance awareness of the cyber, physical, and social contexts of our daily activities. However, the size of image data is much bigger than the scalar data that makes image transmission a challenging issue in batterylimited WVSN. In this paper, we study the energy efficient image aggregation scheme in WVSN. Image aggregation is a possible way to eliminate the redundant portions of the image captured by different data source nodes. Hence, transmission power could be reduced via the image aggregation scheme. However, image aggregation requires image processing that incurs node processing power. Besides the additional energy consumption from node processing, there is another MAC-aware retransmission energy loss from image aggregation. In this paper, we first propose the mathematical model to capture these three factors (image transmission, image processing, and MAC retransmission) in WVSN. Numerical results based on the mathematical model and real WVSN sensor node (i.e., Meerkats node) are performed to optimize the energy consumption tradeoff between image transmission, image processing, and MAC retransmission.
\end{abstract}

\section{Introduction}

1.1. Motivation. The wireless sensor networks (WSNs) are a blooming technology where it can probe and collect environmental information, such as temperature, atmospheric pressure, and irradiation to provide ubiquitous sensing, computing, and communication capabilities. Besides collecting these scalar data (e.g., temperature) from the environment, a newer trend on the WSN is to deploy sensor node with camera to capture and transmit the image data back to the sink node. Thanks to the rapid advancement of sensor technology and the proliferation of consumer electronic devices (e.g., smartphones) equipping the sensors, sensor nodes can send the captured images to provide richer information on sensing and monitoring. This kind of camera-based sensor networks is known as the wireless visual sensor networks (WVSNs). WVSN consists of tiny camera sensor nodes, embedded processor, and wireless transceiver [1].
The WVSN is totally different from traditional WSN in three ways.

(1) New definition of data source nodes: in traditional WSN, when event occurs, the nodes within the sensing range of the event will sense the event and become the data source nodes to transmit the sensed data back to the sink. However, in WVSN, besides the sensing range, one more important criterion, Field of View (FoV), should be considered. The FoV comes from the fact that the camera captures images of the event from a certain direction. In other words, the sensor nodes inside the sensing range could not capture the images of event if the FoV does not cover the event. In Figure 1, we illustrate an example to illustrate the idea of FoV. In Figure 1(a), every sensor node (i.e., nodes $A, B, C, D$, and $E$ ) within the sensing range of the event will sense the data and then transmit the sensed data back to sink. In Figure 1(b), we could observe that even though nodes $D$ and $E$ are within the sensing range, the FoV for these two camera sensors do not 


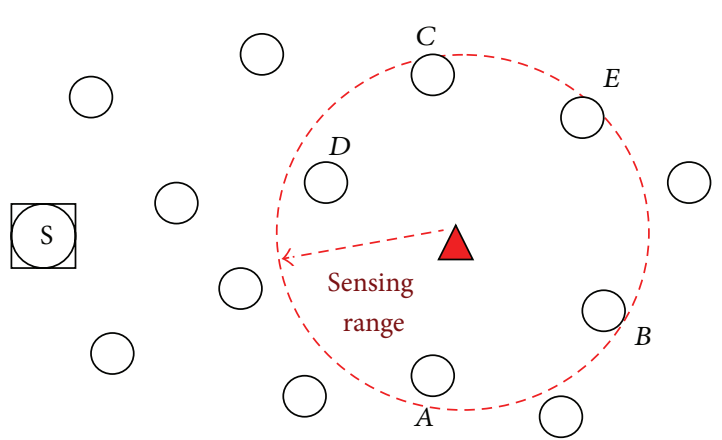

$\triangle$ Event source
(S) Sink node

(a) Traditional WSN

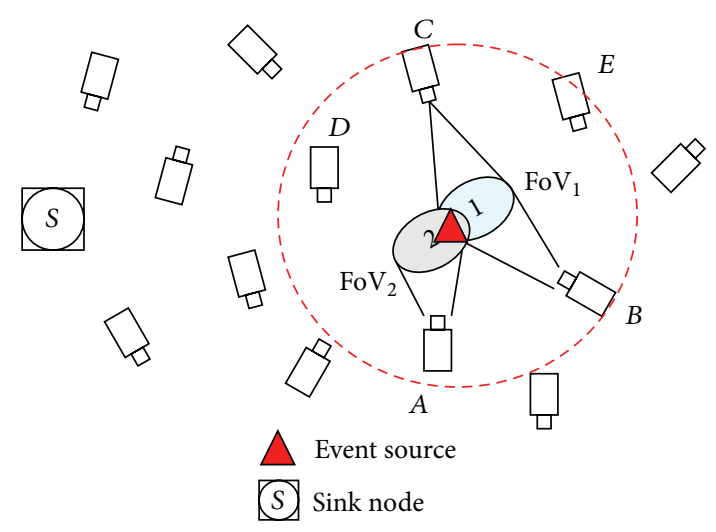

(b) WVSN

FIGURE 1: Wireless sensor networks versus wireless visual sensor networks.

cover the event so that nodes $D$ and $E$ are not the data source nodes. In this case, only nodes $A, B$, and $C$ are the data source nodes that can capture the image of the event and send it back to the sink.

(2) System resource consumption: the image data size is much larger than the scalar data so that it requires larger bandwidth in transmission. Transmit image in wireless WVSN is a challenging issue because the bandwidth and frequency spectrum are limited resources in wireless WVSN. In order not to consume so many system resources, one possible way is to reduce the size of the captured image. Existing image compression or other sophisticated image processing could reduce the image size either via lossy or lossless approach. However, the limited processing power for tiny camera sensor node restricts it from performing complex image processing task. Hence, there is a tradeoff between system resource (bandwidth, frequency spectrum, and processing power) consumption and reducing image size for transmission.

(3) Power-efficient design: due to the fact that most of the sensor nodes are powered by the battery and it is unlikely to exchange battery at the depleted sensor node, powerefficient design is crucial to prolong the life of WSN and WVSN. In WSN, data aggregation scheme [2] could not only combine the data coming from different sources to eliminate the redundancy, but also minimize the total number of transmissions. This makes data aggregation a promising powerefficient data transmission solution. In traditional WSN, the sensed data is a scalar value so that the data combining or computing from multiple sources is easy so that the data processing power on the data aggregation node could be neglected. However, in WVSN, the sensed data is image and it requires sophisticated image processing technique to perform image aggregation so that it incurs significant power consumption at the node processing. Hence, besides the power for image transmission, the power for image processing should also be addressed carefully to give the power-efficient design in WVSN.
Based on the previous discussion, the design problem in WVSN is more challenging than in WSN. Existing algorithms on WSN could not be applicable to the WVSN due to capturing and transmitting image data which are totally different from those in the scalar data. In Figure 2, we give an illustrative example to show the difference between data aggregation and image aggregation. In Figure 2(a), the average temperature is sent to the sink node. After node $A$ receives two temperature values, it will first compute the average value (i.e., 30) and then send it to the sink node. With this data aggregation, node $A$ only needs to send one copy instead of two copies of the temperature value, which could save the energy consumption at node $A$. In Figure 2(b), the images captured at Node $B$ and Node $C$ are sent back to sink node. At Node $A$, it has two choices; one is to send these two images to the sink node and the other one is to combine the two images and then send it to the sink node. In the first approach, Node $A$ needs to transmit two images. In the second approach, Node $A$ needs to perform image processing to combine the two images and send the combined one to the sink node. As compared to computing the averaged temperature value in Figure 2(a), image processing at the relay node $A$ is much more computationally intensive. Hence, the node processing power consumption is nonnegligible for performing the image processing. In other words, the most power-efficient scheme for node $A$ depends on the tradeoff between power saving from transmitting smaller image size and extra power consumption from performing image processing task.

As indicated in Figure 2(b), image aggregation is to remove the redundant part of the received images (i.e., $\left.\left(\mathrm{FoV}_{B} \cap \mathrm{FoV}_{C}\right)\right)$ to give the combined image in smaller size. In the existing image/vision processing literature, several research works propose image comparison methods to combine the images to eliminate the redundant parts in these images [3-5]. Even though the image/vision processing technique is beyond the scope of this paper, we will show the basic idea of these approaches in order to clearly define 


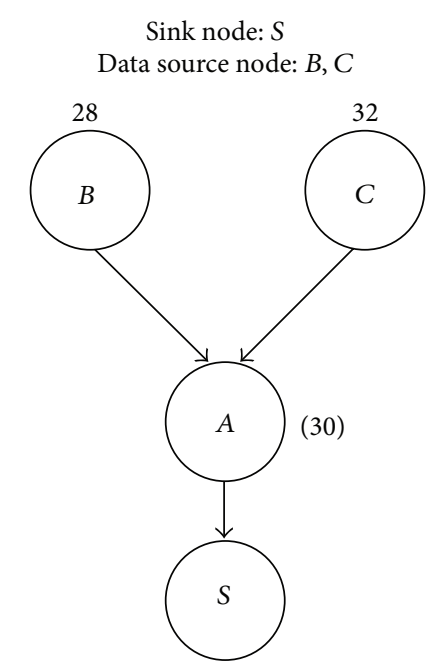

(a) Data aggregation

Sink node: Data source node: $B, C$

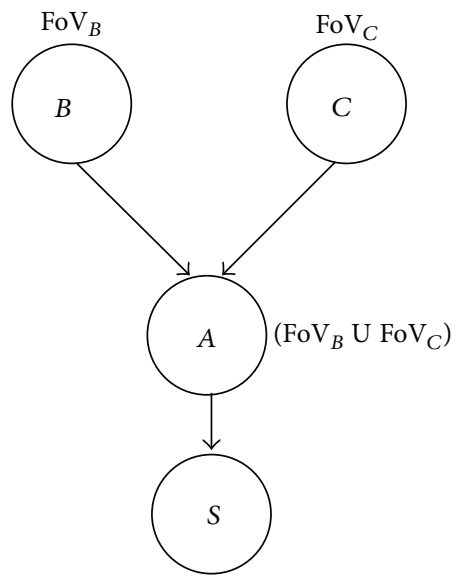

(b) Image aggregation

FIGURE 2: Data aggregation versus image aggregation.

the "image aggregation" in this paper. In [3], they propose the feature-based image comparison methods to compare different images and then find similar image pairs using a set of local corner features from each image. In $[4,5]$, other local features called blob-like feature detectors that are efficient in image comparisons are proposed. These features can be used for feature corner point detection on the raw image. By successfully identifying these features, the similarity between images is measured by the number of matching feature pairs they have.

In the WVSN, when an event occurs, neighboring sensors that are geographically close will sense the event and capture the images. It could be expected that there are many common subregions in images; even the sensors capture the images from different directions or angles. In [6], it states that if two images are from the same planar scene but with different angles, then the two image planes can be related by a homography transform. To be more specific, if the two image planes are related by a homography transform, the corresponding matching feature points are also related by the same homography transform. Based on the theory from [6], the similarity between images could be measured by the number of matching image features and the number of matching image features after homography transform. In other words, when the FoV from the images are the same but with different angle, the features from these images should be the same after a homography transform. For example, in Figure 1(b), Nodes $B$ and $C$ captured the same FoV but with different angle; then the image features from these two images are the same after a homography transform.

Based on the previous discussions on the existing literature on vision/image processing techniques for image comparison, we define the process of image aggregation as follows. If image aggregation is to be performed at the relay node or the sink to combine multiple images, then the aggregated node should first compute the image features for these images and then by using these image features to perform image comparison methods [3-6] to eliminate the redundant (or common) parts of the images. For example, in Figure 2(b), node $A$ has to compute the image features from images $\mathrm{FoV}_{B}$ and $\mathrm{FoV}_{C}$. Then by using the image comparison method as indicated in [3-6], the redundant part (i.e., $\left(\mathrm{FoV}_{B} \cap \mathrm{FoV}_{C}\right)$ ) could be identified so that node $A$ could send the combined image (i.e., $\left(\mathrm{FoV}_{B} \cup \mathrm{FoV}_{C}\right)$ ) with smaller size to the sink node. To be more specific, image aggregation is to have a combined image without the redundant parts, and the redundant parts refer to the overlapped FoV from the captured images.

Note that in the previous discussion, we only address the power consumption at the relay node $A$. When considering the power consumption at the data source nodes (i.e., nodes $B$ and $C$ in Figure 2), besides the power consumption for transmitting the images, the extra energy consumption from retransmission should be also addressed. In wireless networks, because of the hidden node problem, data collision at the receiver side will happen when multiple transmitters transmit the data to the same receiver. When collision occurs, retransmission from the transmitter will be required to ensure that the image could be correctly received [7]. This will incur additional power consumption. Hence, besides the node processing and image transmission power consumption, the extra energy consumption from retransmission should also be considered in the image aggregation. Hence, to capture the energy consumption in WVSN, the interplay of three factors (image transmission, node processing, and retransmission) should be carefully considered. In this paper, for the first time, we propose the mathematical model to consider these three factors and verify them via the numerical results to determine the energy efficient image aggregation scheme.

1.2. Literature Survey. Thanks to the advance of CMOS technology, low power camera could be equipped on sensor nodes. Instead of only probing and sensing the scalar data, sensor nodes equipped with CMOS camera could collect visual data from the environment. As compared to the WSN, 
VSN opens a new sensing function and application era. With this visual data capturing capability, VSN could provide much more information on the monitored environment that makes VSN a promising solution on security surveillance, environment monitoring, and wildlife observation. In VSN, like WSN, most of the sensor nodes are driven by the battery, and it is difficult to replace or replenish the battery for the sensor nodes. Energy-saving transmission has been a hot research issue in WSN, and a lot of research works have proposed energy-saving algorithms and solutions [8] in WSN. However, these traditional energy saving schemes in WSN are not applicable to the VSN due to the particular sensing operations and large captured data size in VSN. Energy efficient transmission strategies in VSN need to address two interdiscipline (WSN and image processing) domains simultaneously.

From the image processing perspective, image compression (e.g., JPEG or JPEG 2000 [9]) has been a popular method to reduce the image size so as to reduce the transmission power. In [10], they develop an energy consumption model for each stage of the JPEG scheme, which can be used to evaluate the JPEG energy dissipation in VSN. Besides image compression, recent works proposed the distributed source coding scheme by the neighboring sensor nodes to deal with the overlapping FoV problem [11]. By using the distributed source coding scheme on the data source sensor nodes with overlapping FoV, the overlapped region of the image could be transmitted only once to save the transmission power. The distributed source coding scheme requires synchronization between the data source node (encoders) so that the sink node (decoder) could use the time-correlated information to decode the received packets $[12,13]$.

From the WSN perspective, data aggregation routing has been an active research topic, and lots of works have proposed novel energy efficient data aggregation routing heuristics [8]. However, as discussed in the previous section, these data aggregation heuristics in WSN are not applicable to the VSN due to the fact that the FoV is different from the traditional sensing range and the image is much larger and more complicated than the scalar data. It could be expected that the image processing is more energy consuming than traditional scalar data aggregation. Most of the recent works in WVSN networks assume that image aggregation, just as the data aggregation in WSN, could save the power $[14,15]$. However, for the Meerkats nodes in [16], they found that the processing time for acquiring and processing image is 2.5 times longer than the time for transmitting the compressed image. In [14], the authors study how to transmit the captured image to the mobile sink node. They assume that image aggregation at the intermediate node to combine the overlapped portions of the captured images could save transmission energy. Under this assumption, multipath routing protocol is proposed to route individual portions of the images to the mobile sink node. Based on the assumption that the transmission task is the most energy consuming one, in [15], they find the best compression ratio to tradeoff between reducing the transmission time, returning objective adequate image quality, and compression time. In [17], they also study the timing on image segmentation and compression for VSN.
The timing selection is either sending data to the central station without further processing or processing locally at the data source node and then transmitting the processed results. They conclude that sending the compressed image after segmentation will be the most energy efficient scheme.

Even though these recent works [14-17] on WVSN networks address the interplay between the image transmission and image processing, they do not consider the MAC retransmission power loss so that it is not applicable to the real WVSN networks. Several works have addressed the MAC-aware data aggregation routing in WSN. In [7], they study the energy consumption tradeoffs between the data aggregation and retransmission in wireless sensor network by using the Carrier Sense Multiple Access/Collision Avoidance (CSMA/CA) MAC protocol. In [18], they propose an MAC Anycasting to achieve spatial convergence and Randomized Waiting protocol to achieve the temporal convergence. These spatial convergence and temporal convergence properties maximize the advantages of data aggregation in structurefree (i.e., no preconstructed network structure) wireless sensor networks. To the best of our knowledge, there is no existing literature addressing the MAC retransmission problem in the WVSN networks. In this paper, for the first time, we consider the interplay between the image transmission, image processing, and MAC retransmission to identify the energy efficient image aggregation scheme in WVSN networks.

The remainder of this paper is organized as follows. In Section 2, we propose the mathematical model of the image aggregation scheme to capture the power consumption for the image transmission, image processing and MAC retransmission. In Section 3, numerical results based on the mathematical model and real WVSN sensor nodes are conducted to identify the best image aggregation strategy in different network settings. Finally, Section 4 concludes this paper.

\section{Mathematical Model for Image Transmission in WVSN}

The WVSN is modeled as a graph in which sensors are represented as nodes and the arc connecting two nodes indicates that one sensor is within the other's transmission radius. In Section 2.1, we will study the node processing and transmission power consumption at the relay node. In Section 2.2, besides the power consumption at the relay node, we will also study the retransmission power consumption at the data source nodes.

\subsection{Image Aggregation Power Consumption Model (Retrans-} mission Power Is Not Covered). Before giving the general image aggregation power consumption model, the power consumption from retransmission is not considered to get a clear picture of the tradeoff between node processing and image aggregation. We first consider the image aggregation case in Figure 2(b). In this case, Node $A$ receives two images and then transmits the combined image to the sink node. The power consumption for Node $A$ includes the image processing power and the image transmission power. Note 
that total power consumption for Node $A$ also includes the image receiving power. The image receiving power is all the same regardless of using the image aggregation or not. Therefore, the image receiving power is not included in the following model so that we could focus on studying the tradeoff of image aggregation on image processing power and image transmission power.

Before deriving the model, we first give the notations used in the formulation, as indicated in Table 1.

In Figure 2(b), the captured image sizes of Node $B$ and Node $C$ are $\left|F_{B}\right|$ and $\left|F_{C} V_{C}\right|$. Then the received image size for Node $A$ is $\left(\left|F_{B} V_{B}\right|+\left|F_{C}\right|\right)$. If Node $A$ performs image aggregation, the size of the combined image is $\left|\left(F_{0} V_{B} \cup F_{C} V_{C}\right)\right|$. In this case, the image processing power for Node $A$ is $\Phi_{A}\left(\left|F_{B} V_{B}\right|+\left|F_{C}\right|,\left|\left(F_{B} V_{B} \cup F_{C}\right)\right|\right)$. And the transmission power for Node $A$ is $\Psi_{A}\left(r_{A},\left|\left(F_{B} \cup V_{B} \cup V_{C}\right)\right|\right)$. Let $T_{A 2}$ denote the power consumption for Node $A$ in the image aggregation scheme:

$$
\begin{aligned}
T_{A 2}= & \Phi_{A}\left(\left|\mathrm{FoV}_{B}\right|+\left|\mathrm{FoV}_{C}\right|,\left|\left(\mathrm{FoV}_{B} \bigcup \mathrm{FoV}_{C}\right)\right|\right) \\
& +\Psi_{A}\left(r_{A},\left|\left(\mathrm{FoV}_{B} \bigcup \mathrm{FoV}_{C}\right)\right|\right)
\end{aligned}
$$

If we do not perform image aggregation at Node $A$ in Figure 2(b), there is no image processing at Node $A$. Hence, the image processing power at Node $A$ is zero. In addition, the transmission power for Node $A$ is $\Psi_{A}\left(r_{A},\left|F_{B}\right|+\left|F_{C} V_{C}\right|\right)$. Let $\overline{T_{A 2}}$ denote the power consumption for Node $A$ without the image aggregation scheme:

$$
\overline{T_{A 2}}=\Psi_{A}\left(r_{A},\left|\mathrm{FoV}_{B}\right|+\left|\mathrm{FoV}_{C}\right|\right) .
$$

It is trivial that the image size after combining two images should not be larger than the sum of two images. Hence,

$$
\left.\left|\left(\operatorname{FoV}_{B} \bigcup \mathrm{FoV}_{C}\right)\right|\right) \leq\left|\mathrm{FoV}_{B}\right|+\left|\mathrm{FoV}_{C}\right|
$$

Then we have

$$
\Psi_{A}\left(r_{A},\left|\left(\mathrm{FoV}_{B} \bigcup \mathrm{FoV}_{C}\right)\right|\right) \leq \Psi_{A}\left(r_{A},\left|\mathrm{FoV}_{B}\right|+\left|\mathrm{FoV}_{C}\right|\right) .
$$

From (4), we could only be sure that the transmission power for the image aggregation scheme is better than the nonimage aggregation one. However, we could not conclude that $T_{A 2} \leq \overline{T_{A 2}}$ because there is a nonzero image processing power consumption at $T_{A 2}$. In other words, in Figure 2, image aggregation scheme could reduce the energy consumption only when

$$
\begin{aligned}
& \Phi_{A}\left(\left|\mathrm{FoV}_{B}\right|+\left|\mathrm{FoV}_{C}\right|,\left|\left(\mathrm{FoV}_{B} \bigcup \mathrm{FoV}_{C}\right)\right|\right) \\
& \quad+\Psi_{A}\left(r_{A},\left|\left(\mathrm{FoV}_{B} \bigcup \mathrm{FoV}_{C}\right)\right|\right) \leq \Psi_{A}\left(r_{A},\left|\mathrm{FoV}_{B}\right|+\left|\mathrm{FoV}_{C}\right|\right) .
\end{aligned}
$$

We next generalize (5) to consider $m$ transmitters in Figures 3(a) and 3(b).

In Figure 3(b), the size of total received images is $\sum_{i=1}^{m}\left|\mathrm{FoV}_{i}\right|$ and the size of transmitted aggregated
TABLE 1

\begin{tabular}{ll}
\hline$N$ & The set of all sensor nodes \\
$r_{n}$ & $\begin{array}{l}\text { Transmission radius of the node } n \\
C_{n}\end{array}$ \\
$D_{n}$ & $\begin{array}{l}\text { Received image size for sensor node } n \\
\text { Transmitted image size for sensor node } n\end{array}$ \\
$\Psi_{n}\left(r_{n}, D_{n}\right)$ & $\begin{array}{l}\text { Transmission power function of the node } n, \\
\text { which is a function of the sensor's transmission } \\
\text { radius } r_{n} \text { and transmitted image size } D_{n}\end{array}$ \\
$\Phi_{n}\left(C_{n}, D_{n}\right)$ & $\begin{array}{l}\text { Computing power function of the node } n \text {, which } \\
\text { is a function of the sensor's received image size } C_{n} \\
\text { and transmitted image size } D_{n}\end{array}$ \\
\hline
\end{tabular}

image is $\left(\left|F_{0 V} \cup F_{1} V_{2} \cup \cdots \cup F_{m} V_{m}\right|\right)$. Then the image processing power for Node $A$ is $\Phi_{A}\left(\sum_{i=1}^{m}\left|\mathrm{FoV}_{i}\right|,\left(\mid \mathrm{FoV}_{1} \cup\right.\right.$ $\left.\left.\mathrm{FoV}_{2} \cup \cdots \cup \mathrm{FoV}_{m} \mid\right)\right)$. The transmission power for Node $A$ is $\Psi_{A}\left(r_{A},\left(\left|\mathrm{FoV}_{1} \cup \mathrm{FoV}_{2} \cup \cdots \cup \mathrm{FoV}_{m}\right|\right)\right)$. Let $T_{A}$ denote the power consumption for Node $A$ in the image aggregation scheme:

$$
\begin{aligned}
T_{A}= & \Phi_{A}\left(\sum_{i=1}^{m}\left|\mathrm{FoV}_{i}\right|,\left(\left|\mathrm{FoV}_{1} \bigcup \mathrm{FoV}_{2} \bigcup \cdots \bigcup \mathrm{FoV}_{m}\right|\right)\right) \\
& +\Psi_{A}\left(r_{A},\left(\left|\mathrm{FoV}_{1} \bigcup \mathrm{FoV}_{2} \bigcup \cdots \bigcup \mathrm{FoV}_{m}\right|\right)\right) .
\end{aligned}
$$

If Node $A$ does not perform image aggregation (i.e., Figure 3(a)), then the image processing power for Node $A$ is zero and the transmission power for Node $A$ is $\Psi_{A}\left(r_{A},\left(\sum_{i=1}^{m}\left|\mathrm{FoV}_{i}\right|\right)\right)$. Let $\overline{T_{A}}$ denote the power consumption for Node $A$ without the image aggregation scheme:

$$
\overline{T_{A}}=\Psi_{A}\left(r_{A},\left(\sum_{i=1}^{m}\left|\mathrm{FoV}_{i}\right|\right)\right) \text {. }
$$

Image aggregation scheme is more energy efficient than the nonimage aggregation scheme only when

$$
\begin{aligned}
& \Phi_{A}\left(\sum_{i=1}^{m}\left|\mathrm{FoV}_{i}\right|,\left(\left|\mathrm{FoV}_{1} \bigcup \mathrm{FoV}_{2} \bigcup \cdots \bigcup \mathrm{FoV}_{m}\right|\right)\right) \\
& +\Psi_{A}\left(r_{A},\left(\left|\mathrm{FoV}_{1} \bigcup \cdots \bigcup \mathrm{FoV}_{m}\right|\right)\right) \\
& \leq \Psi_{A}\left(r_{A},\left(\sum_{i=1}^{m}\left|\mathrm{FoV}_{i}\right|\right)\right) .
\end{aligned}
$$

Based on (8), image aggregation scheme is a better choice only when the gain from transmitting smaller aggregated image is greater than the extra energy consumption from performing image processing.

2.2. General Image Aggregation Power Consumption Model. In Section 2.1, we only consider the node processing power and transmission power at the relay node $A$. We do not consider the transmission power for the data source nodes $1,2, \ldots, m$. To capture the whole picture of the power consumption in WVSN, we need to consider the power 


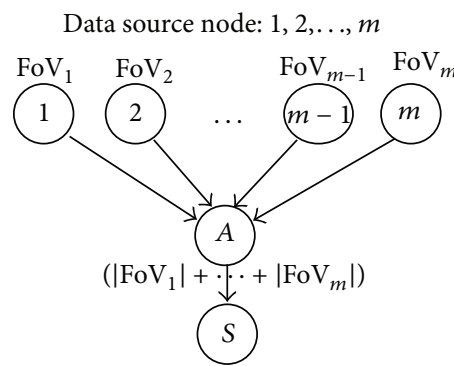

(a) Single relay node (without image aggregation)

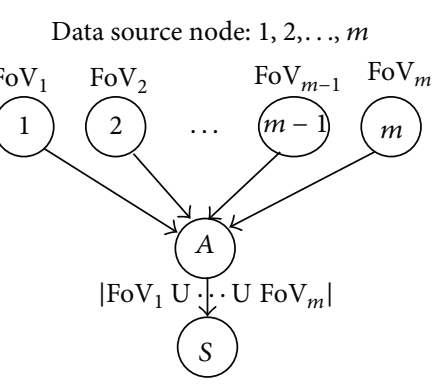

(b) Single relay node (with image aggregation)

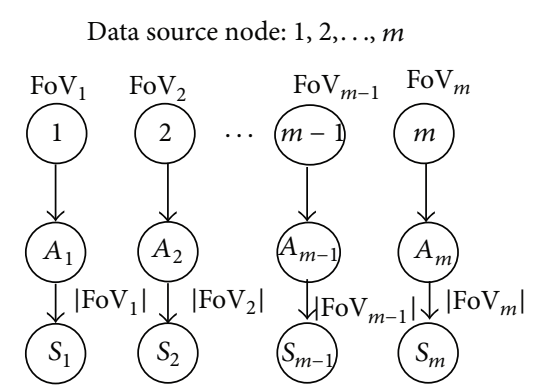

(c) Individual relay node

FIGURE 3: Nonaggregation versus one-hop aggregation.

consumption at the data source nodes. The power consumption in the data source nodes does not only include the transmission power to transmit the image packet but also include the MAC layer retransmission power due to collision.

The analysis of retransmission power is conducted as follows. First of all, we assume that each sensor node is equipped with a CSMA/CA compatible transceiver. We examine the contention-based CSMA/CA protocol-distributed coordination function (DCF). For the DCF, the turnaround time of each successful transmission is from RTS to the contention window. When a sender wants to transmit data to receiver, it will first issue as RTS packet to ask for the transmit permission from the receiver. If permission is granted, the receiver will issue a CTS back to the sender, and then the data could be transmitted. After the data is successfully received, the receiver will transmit an ACK back to the sender. Because of this RTS and CTS mechanism, the hidden node problem will be circumvented. Note that the time interval between RTS and CTS is not larger than short interframe spacing (SIFS) time according to the CSMA/CA protocol. At the end of this round of transmission, all the sensor nodes will contend for the channel after a DIFS period. In order not to interrupt each round of transmission, the DIFS period is larger than the SIFS period. Letting the propagation delay from the sender to the receiver be $\theta$, then the turnaround time is $2 \theta$. Then the overall contention period is $(\mathrm{RTS}+\mathrm{SIFS}+2 \theta)$ for sending the RTS frame. Other sensor nodes hear that the RTS or CTS packet will not transmit to prevent collision in this contention period.

Before giving the mathematical model to consider the retransmission power in WVSN, we fist give the notation used in the formulation, as indicated in Table 2.

Based on the analysis in [19], we derive the mean retransmission time of a sender. We assume that each transmission conforms to geometric distribution and each sensor node generates image packets that follow Poisson distribution with a certain rate, $\lambda$. Successful transmission of image from a sender to a receiver is determined by the number of sensor nodes whose transmission radius covers the receiver. By considering the receiver side collisions in terms of the

\section{TABLE 2}

\begin{tabular}{|c|c|}
\hline$E_{\mathrm{MAC}}$ & $\begin{array}{l}\text { Total retransmission power consumption due to } \\
\text { collision in the MAC layer }\end{array}$ \\
\hline$T_{\text {image }}$ & Transmission time for transmitting an image packet \\
\hline$\underline{\mathrm{RTS}}$ & Transmission time for RTS frame \\
\hline SIFS & Short interframe space time \\
\hline$e_{n}\left(r_{n}\right)$ & $\begin{array}{l}\text { Energy consumption function of node } n \text { per unit time, } \\
\text { which is a function of sensor's transmission radius }\end{array}$ \\
\hline$\Theta$ & $\begin{array}{l}\text { Maximum propagation delay for transmitting data } \\
\text { packet }\end{array}$ \\
\hline$R_{n}$ & $\begin{array}{l}\text { The set of all possible transmission radii that the node } n \\
\text { can adopt, which is a discrete set }\end{array}$ \\
\hline$z_{n k}$ & $\begin{array}{l}=1 \text { if the node } k \text { is covered within transmission radius } \\
\text { of the node } n \text { and }=0 \text { otherwise }\end{array}$ \\
\hline$c_{n k}$ & $\begin{array}{l}\text { The average retransmission times of the node } n \text { to } \\
\text { transmit data to the node } k\end{array}$ \\
\hline
\end{tabular}

communication radius of sensor nodes, the hidden terminal problem is also implicitly contemplated.

In CSMA/CA protocol, when sender wants to transmit a packet to the receiver, it will first issue the RTS control frame and then wait for the CTS frame from the receiver to make sure if the channel is free or not [19]. If there is no other transmitter transmitting a packet within this contention period, then this transmission is successful. We then derive the average retransmission times of the node $n$ to transmit data to the node $k$ (denoted as $c_{n k}$ ):

$$
c_{n k}=\frac{1}{p_{\text {success }(n, k)}}=\frac{1}{e^{-\lambda(\underline{\mathrm{RTS}}+\underline{\mathrm{SIFS}}+2 \theta) \sum_{j \in N, j \neq n} z_{j k}} .}
$$

The meaning of the previous retransmission function is the mean value of the geometric distribution where the successful transmission probability, say $p_{\text {success }}$, is that no data transmission is occurring at any node whose transmission radius covers receiver node $k$ within the interval of (RTS $+\underline{\text { SIFS }}+$ $2 \theta$ ). Using $z_{j k}$ we can calculate the total number of sensor 
nodes whose transmission covers sensor node $k$ or the total number of sensor nodes covered by transmission radius of sensor node $j$. We illustrate an example in Figures 2(b) and 2(c) to calculate the value of retransmission times. For any node $n \in\{1, \ldots, m\}, \sum_{j \in N, j \neq n} z_{j k}=(m-1)$, then

$$
c_{n k}=\frac{1}{p_{\text {success }(n, k)}}=\frac{1}{e^{-\lambda(\underline{\mathrm{RTS}}+\underline{\mathrm{IFS}}+2 \theta)(m-1)}} .
$$

Based on (10), we can calculate the total power consumption with consideration of the MAC layer retransmission. Note that the retransmission power consumption comes from collision at the receiver, so if the transmission to the receiver is only from one transmitter, then there is no retransmission power consumption. Hence, in Figures 3(a) and 3(b), because there are $m$ transmitters that transmit the image data to the node $A$, there might be collision at node $A$ to incur retransmission at the data source node. However, in Figure 3(c), since the receiver receives the transmission from one transmitter, there will be no retransmission power loss from collision. In (11), we show the MAC retransmission power consumption for the data source nodes that are aggregated at node $A$ :

$$
E_{\mathrm{MAC}}=(\underline{\mathrm{RTS}}) \cdot\left(\sum_{n \in\{1, \ldots, m\}} c_{n A} \cdot e_{n}\left(r_{n}\right)\right) .
$$

In (11), it is stated that the MAC retransmission power is equal to the power of transmitting the RTS frame multiplied by the number of retransmission times. By substituting (10) with (11), we have

$$
\begin{aligned}
E_{\mathrm{MAC}} & =(\underline{\mathrm{RTS}}) \cdot\left(\sum_{n \in\{1, \ldots, m\}} \frac{1}{e^{-\lambda(\underline{\mathrm{RTS}}+\underline{\underline{\mathrm{SISS}}}+2 \theta)(m-1)}} \cdot e_{n}\left(r_{n}\right)\right) \\
& =(\underline{\mathrm{RTS}}) \cdot\left(\frac{1}{e^{-\lambda(\underline{\mathrm{RTS}} \underline{\underline{S I F S}}+2 \theta)(m-1)}}\right) \cdot\left(\sum_{n \in\{1, \ldots, m\}} e_{n}\left(r_{n}\right)\right) .
\end{aligned}
$$

Note that there is no MAC retransmission power consumption for Figure 3(c) due to the fact that each data source node transmits the image to different relay node. Hence, in considering the total power consumption, only Figures 3(a) and 3(b) need to address (12). In the following, we derive the total power consumption for "Nonaggregation" scheme in Figure 3(a) and "Aggregation" scheme in Figure 3(b) and "Individual relay" scheme in Figure 3(c), each denoted as the $\operatorname{Total}_{a}$, Total $_{b}$, and Total ${ }_{c}$, respectively,

$$
\begin{aligned}
\operatorname{Total}_{a}= & E_{\mathrm{MAC}}+\left(T_{\text {image }}\right) \cdot\left(\sum_{n \in\{1, \ldots, m\}} e_{n}\left(r_{n}\right)\right) \\
& +\Psi_{A}\left(r_{A},\left(\sum_{i=1}^{m}\left|\mathrm{FoV}_{i}\right|\right)\right),
\end{aligned}
$$

$$
\begin{aligned}
\operatorname{Total}_{b}= & E_{\mathrm{MAC}}+\left(T_{\text {image }}\right) \cdot\left(\sum_{n \in\{1, \ldots, m\}} e_{n}\left(r_{n}\right)\right) \\
& +\Phi_{A}\left(\sum_{i=1}^{m}\left|\mathrm{FoV}_{i}\right|,\left(\left|\mathrm{FoV}_{1} \bigcup \ldots \bigcup \mathrm{FoV}_{m}\right|\right)\right) \\
& +\Psi_{A}\left(r_{A},\left(\left|\mathrm{FoV}_{1} \bigcup \ldots \bigcup \mathrm{FoV}_{m}\right|\right)\right), \\
\operatorname{Total}_{c}= & \left(T_{\text {image }}\right) \cdot\left(\sum_{n \in\{1, \ldots, m\}} e_{n}\left(r_{n}\right)\right) \\
& +\sum_{i=1}^{m} \Psi_{A_{i}}\left(r_{A_{i}},\left|\mathrm{FoV}_{i}\right|\right) .
\end{aligned}
$$

In (15), there is no MAC retransmission power consumption since each data source node transmits the image to individual relay node. In addition, there is no node processing power at (14) and (15). In the following section, we will verify which scheme is the most energy efficient one.

\section{Numerical Results}

We assume that a sensor network operates in event-driven mode. When there is an event, the image sensor nodes that could capture the images of the event (i.e., the images nodes whose FoV cover the event) become the data source nodes and send the images back to the sink node. In the experiments, the parameter setting for the CSMA/CA parameters (i.e., RTS, CTS, $\Theta$ ) is based on [7].

In the first set of experiments, we study the total power consumption comparison among these three image aggregation routing schemes as indicated in Figure 3. The power consumption is normalized with respect to the "Individual relay" scheme. Hence, the "relative power consumption" $=X /$ (the power consumption from Individual relay scheme), where $X$ is the power consumption from the other scheme. For example, if the power consumption for the "Nonaggregation" scheme is 1.5 times of the "Individual relay" scheme, then the relative power consumption for the "Nonaggregation" scheme is 1.5 .

The power consumption values for the image transmission (i.e., $\left.\Psi_{n}\left(r_{n}, D_{n}\right)\right)$ and image processing (i.e., $\Psi_{n}\left(r_{n}\right.$, $\left.D_{n}\right)$ ) are very important parameters in the experiments. We simulate the five different power ratios between the image transmission and the image processing (i.e., $\left.\left(\Psi_{n}\left(r_{n}, D_{n}\right) / \Phi_{n}\left(C_{n}, D_{n}\right)\right)\right)$, ranging from 0.55 to 4.55 with step size 1 to examine the solution quality for three image aggregation schemes. We denote such ratio as the $T / P$ ratio. To be more specific, we fix the value of the image transmission and change the value of image processing (i.e., five different values) in the experiments. As could be observed from (13) (15), among these three image aggregation schemes, the image processing power only exists in the "Aggregation" scheme. It could be expected that the power consumption for the "Individual relay" and "Nonaggregation" will remain the same in different $T / P$ ratio. On the other hand, the "Aggregation" scheme 


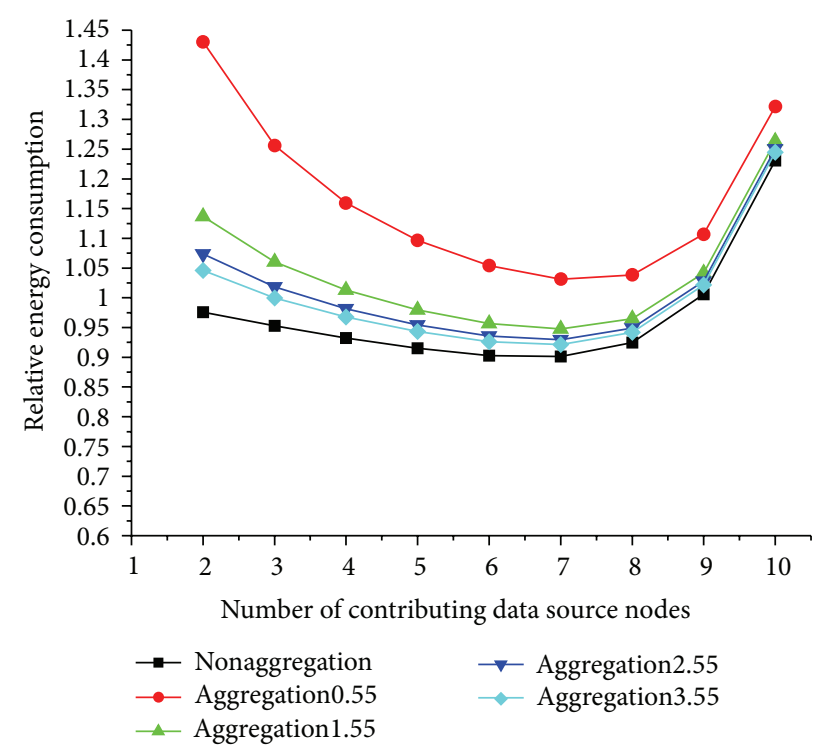

FIGURE 4: Energy consumption comparison in $0 \%$ of aggregation.

has different relative power consumption with respect to different $T / P$ ratio. "Aggregation 1.55 " means the relative power consumption when the $T / P$ ratio is 1.55 . Note that we do not plot the "Aggregation 4.55" in the figure due to the fact that the value of "Aggregation 4.55 " is too close to the value of "Aggregation 3.55 ".

From Figures 4, 5, 6, 7, 8, and 9, we show the performance comparison with respect to the degree of aggregation. $Y \%$ aggregation means that $Y \%$ of the image size captured by the data source nodes is the same and could be aggregated to be transmitted to the sink node in one copy. Hence, the other $(100-Y) \%$ of the image captured by each data source node is different. For example, assuming that the image size is $48 \mathrm{~KB}$, then when there are three data source nodes and $20 \%$ aggregation, by using the Aggregation scheme, the total image size transmitted to the sink node is $48 \mathrm{~KB} \times(1+(3-$ $1) \times(1-0.2))=124.8 \mathrm{~KB}$. And the total image size would be $48 \mathrm{~KB} \times(1+(3-1) \times(1-0.8))=67.2 \mathrm{~KB}$. Note that by using the other two schemes (i.e., Nonaggregation and Individual), the total image size transmitted to the sink node is $48 \mathrm{~KB} \times$ $3=144 \mathrm{~KB}$. Hence, in high degree of aggregation, we could expect a low transmitted image size for the "Aggregation" scheme.

From Figure 4 to Figure 9, there are three important observations.

(1) "Aggregation" scheme performs better when in higher $T / P$ ratio. From Figure 4 to Figure 9, we could observe that the value of "Aggregation $X$ " is lower than the "Aggregation $Y$ " when $X>Y$. In other words, by increasing the $T / P$ ratio, the saving from image transmission could be more significant than the power consumption from the image processing so that the total power consumption could be reduced. In addition, we could see there is a big gap between "Aggregation 0.55 " and "Aggregation1.05". At "Aggregation 0.55", the image processing power is larger than the image transmission power. In this case, performing the image aggregation is

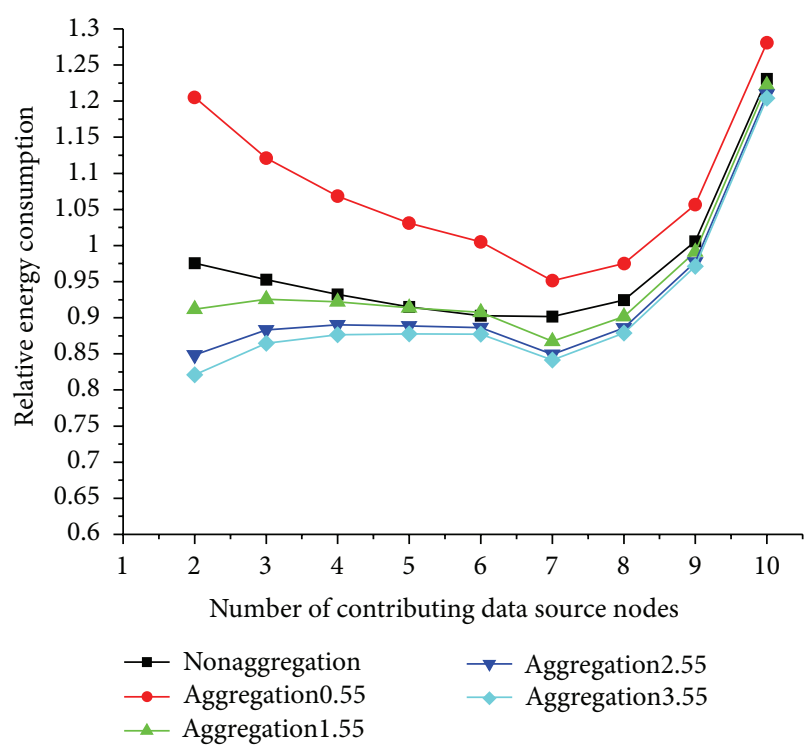

FIGURE 5: Energy consumption comparison in 20\% of aggregation.

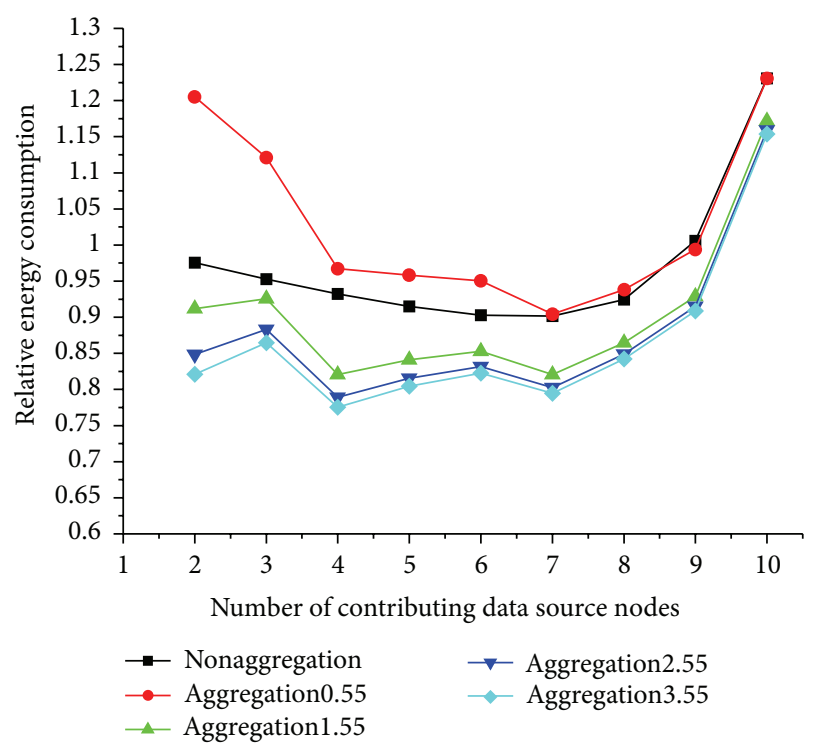

FIGURE 6: Energy consumption comparison in 40\% of aggregation.

not a good choice especially in lower degree of aggregation. When the degree of aggregation is less than $40 \%$, the power consumption for "Nonaggregation" is even lower than the "Aggregation 0.55 ". On the other hand, when $T / P$ ratio is greater than 1 , performing the image aggregation is encouraged to decrease the total power consumption especially in higher degree of aggregation.

(2) "Aggregation" scheme performs better when in higher degree of aggregation: when in higher degree of aggregation, the size of the aggregated image will be smaller for the "Aggregation" scheme. Then the transmission power will be lower to transmit the aggregate image. Note that the image processing power is almost the same regardless of the degree of aggregation. Then the "Aggregation" scheme performs 


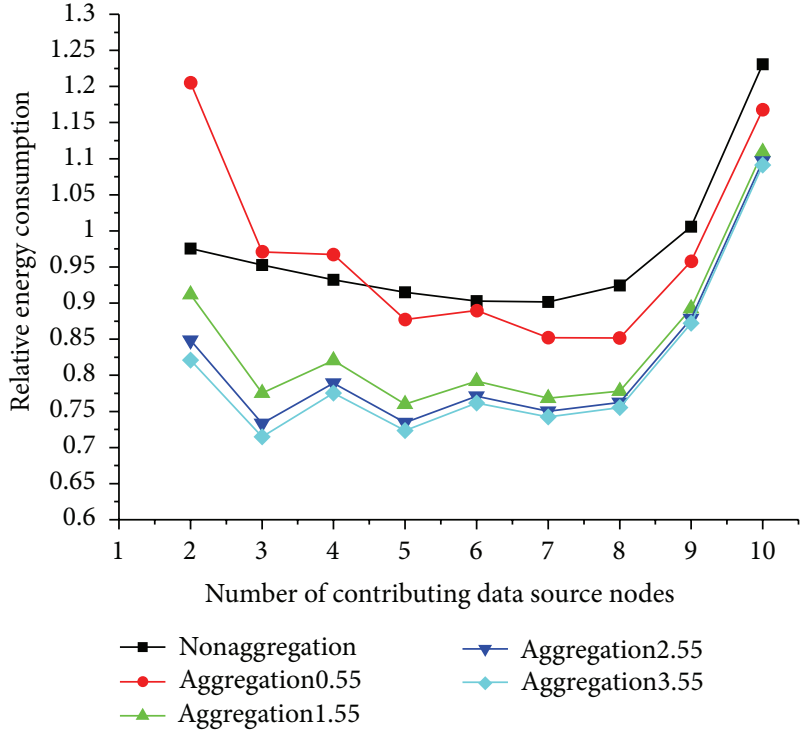

FIGURE 7: Energy consumption comparison in $60 \%$ of aggregation.

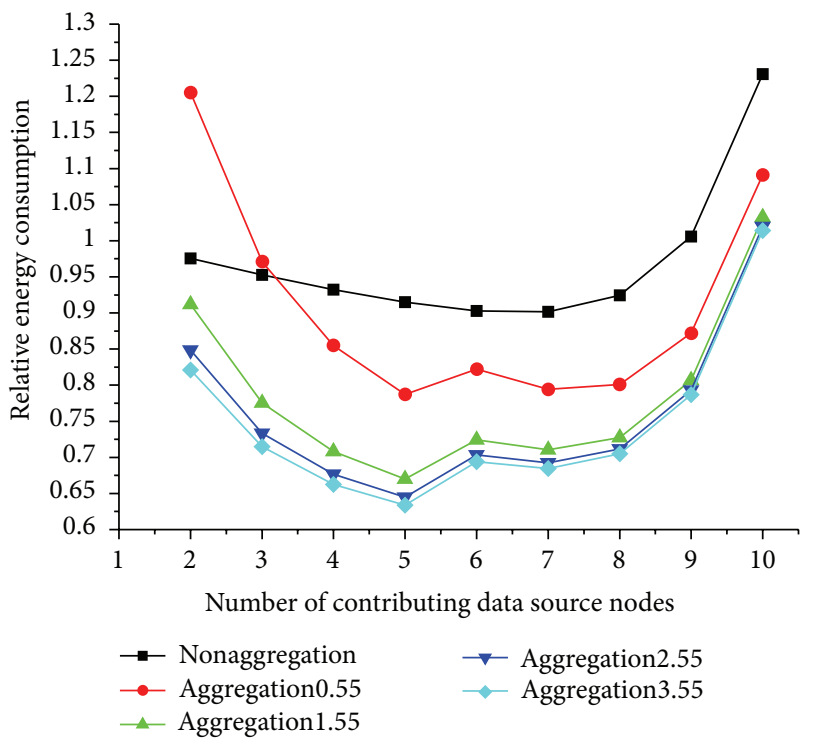

FIGURE 8: Energy consumption comparison in $80 \%$ of aggregation.

better in higher degree of aggregation. In Figure 4, when in $0 \%$ of aggregation (i.e., the captured images from the data source node are all different), the "Aggregation" scheme could only achieve $90 \%$ of power consumption with respect to the "Individual relay" scheme. In Figure 9, when in 100\% of aggregation (i.e., all the captured images from the data source nodes are identical), the "Aggregation" scheme could achieve $60 \%$ of power consumption with respect to the "Individual relay" scheme.

(3) Increasing the number of data source nodes also increases the multiplexing gain and the MAC retransmission power consumption. From Figure 4 to Figure 9, we could see that there is a very interesting " $U$ " shape relative power consumption for "Nonaggregation" and "Aggregation" schemes

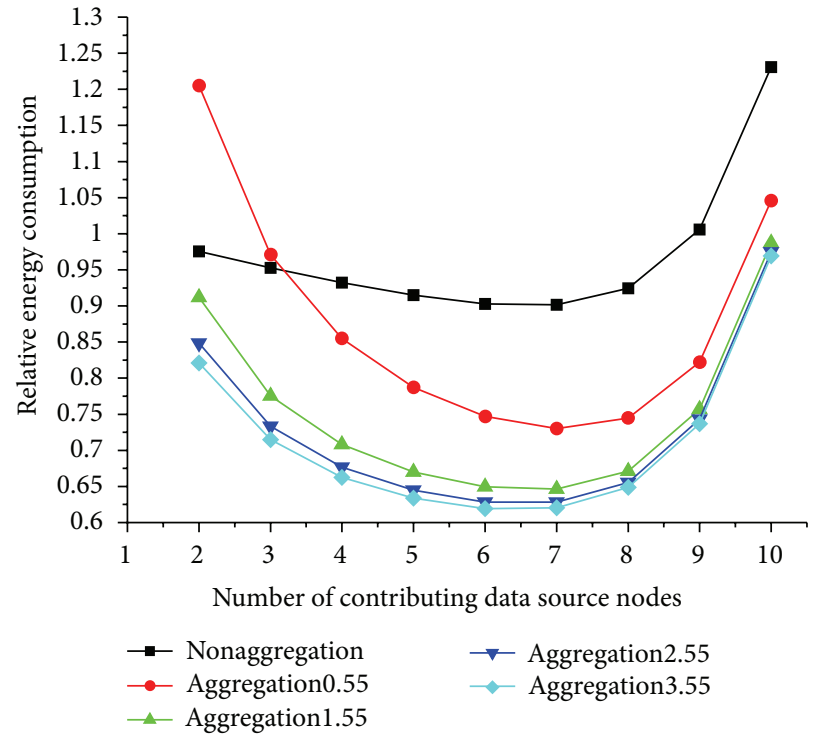

FIGURE 9: Energy consumption comparison in $100 \%$ of aggregation.

with respect to the number of data source nodes. Intuitively, increasing the number of data source nodes will increase the multiplexing gains from aggregating multiple images to the single relay node. Hence, we could expect that the relative power consumption for "Nonaggregation" and "Aggregation" schemes will keep decreasing when increasing the number of data source nodes. However, we could observe that the relative power consumption for these two schemes will be first decreasing and then increasing. The reason for increasing is because of the MAC retransmission power consumption. Note that as the number of data source nodes increases, it also increases the collision probability at the relay node so that it increases the retransmission power consumption. From Figure 4 to Figure 9, we observe that before seven data source nodes, the multiplexing gain will dominate the MAC retransmission power consumption. However, after seven data source nodes, the MAC retransmission power consumption will dominate the multiplexing gain so that the relative power consumption will start increasing. This gives the interesting " $U$ " shape relative power consumption for "Nonaggregation" and "Aggregation" with respect to the number of data source nodes.

In the first set of experiments, the power consumption experiments are based on the derived mathematical model. In the second set of experiments, in order to get the real power consumption for image processing and transmission in WVSN, we use the incremental charge drawn on the board of the Meerkats nodes [16] as shown in Table 3. The voltage for the battery used in Meerkats node is 7.4 Volt. Because the energy consumption is equal to the charge drawn times the battery voltage, therefore, the third column of Table 3 is equal to the second column of Table 3 multiplied by the Meerkats battery voltage (i.e., 7.4 Volt). In the first set of experiments, we could observe that in most of the cases, "Nonaggregation" and "Aggregation" schemes outperform the "Individual relay" scheme. In the second set of experiments, we will only address 


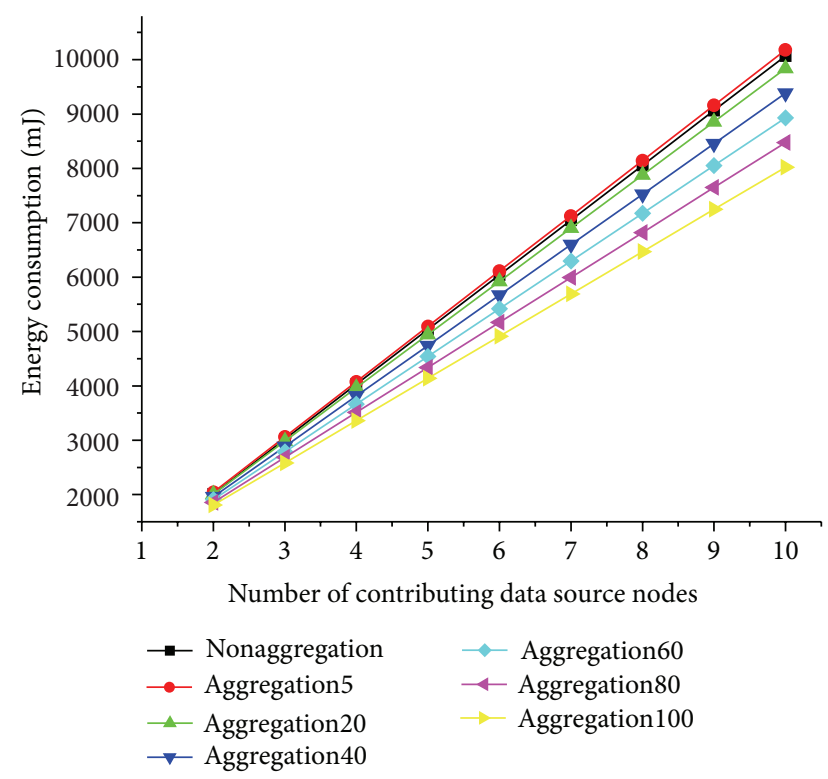

FIGURE 10: Energy consumption comparison with degree of aggregation.

TABLE 3: Incremental charge drawn (in milli-Coulombs) and energy consumption (in milli-Joules) on Meerkats nodes.

\begin{tabular}{lcc}
\hline Task & $\begin{array}{c}\text { Incremental } \\
\text { charges } \\
(\mathrm{mC})\end{array}$ & $\begin{array}{c}\text { Energy } \\
\text { consumption } \\
(\mathrm{mJ})\end{array}$ \\
\hline $\begin{array}{l}\text { Acquire and compress image } \\
\begin{array}{l}\text { Acquire, process, and compress image } \\
\text { portion (event detected) }\end{array}\end{array}$ & 102 & 755 \\
Send 48 KB (full compressed image) & 34 & 777 \\
\hline
\end{tabular}

the comparison between the "Nonaggregation" and "Aggregation" schemes. Since these two schemes incur the same MAC retransmission power consumption, in the following experiments, we will only study the image transmission and image processing power consumption comparison between these two schemes.

In the following experiments, the "Aggregation $X$ " means that $X \%$ of the image size captured by the data source nodes is the same and could be aggregated to be transmitted to the sink node in one copy. Hence, the other $(100-X) \%$ of the image captured by each data source node is different and should be transmitted to the sink node individually. In Figure 10, there are two important observations.

(1) Aggregation lowers the energy consumption. We can observe that as the percentage of the same image increased, aggregation scheme could keep lowering the energy consumption. In Figure 10, Aggregation5 scheme consumes the largest energy and Aggregation100 consumes the least energy. This is because in the Aggregation100, the image captured by each data source node is the same so that only one copy of the image is transmitted to the sink node.
(2) Nonaggregation scheme consumes more energy after the portion of the same image is more than $10 \%$. In Figure 10, we could observe that Aggregation5 scheme consumes more energy than Nonaggregation scheme. Nonaggregation scheme consumes almost the same amount of energy as the Aggregation10 scheme. After the portion of same image is more than $10 \%$, aggregation scheme is more energy efficient than the Nonaggregation scheme.

In the next experiment, we study the energy comparison with respect to the distance. We assume that the attenuation factor is 2. Hence, the transmission power consumption is proportional to the transmission radius to the power of 2. Letting the transmission radius used in Table 3 be $r$, then for transmission radius $k \times r$, the transmission energy consumption would be $252 \times k^{2} \mathrm{~mJ}$. For example, when the transmission radius is $2 r$, the energy consumption will be $252 \times 4=1008 \mathrm{~mJ}$. Note that changing the distance between the transmitter and receiver does not affect the image processing at the node, so the energy consumption at step 1 and step 2 in Table 3 remains the same. Only the transmission power for step 3 in Table would be changed. Recall that in the first set of experiments, we simulate the performance of aggregation with respect to the $T / P$ ratio. Increasing the transmission radius for the Meerkats nodes is equivalent to increasing the $T / P$ ratio. We try to verify if the conclusion for the image aggregation with respect to the $T / P$ ratio in the first set of experiments is still valid for the real Meerkats nodes.

In order to compare the efficiency of data aggregation, we devise a relative energy consumption index:

$$
\mathrm{REI}=\left(\frac{\text { Aggregation } X}{\text { Nonaggregation }}\right) .
$$

If REI is greater than 1, it means that the AggregationX scheme consumes more energy than the Nonaggregation scheme. On the other hand, AggregationX scheme is more energy efficient than the Nonaggregation scheme when REI is smaller than 1 .

From Figure 11 to Figure 13, we have three important observations.

(1) REI becomes smaller as the transmission radius becomes larger. When the transmission radius becomes larger, the transmission power will be increased. In this case, without aggregation, the Nonaggregation scheme has to transmit more copies of the images than the Aggregation $X$ scheme so that the Nonaggregation scheme will consume more energy than the other Aggregation $X$ schemes. Hence, REI will be smaller as the transmission radius becomes larger.

(2) Aggregation lowers the REI. We can observe that as the percentage of the same image increased, aggregation scheme could keep lowering the REI. From Figure 11 to Figure 13, the REI for the Aggregation10 scheme is the largest and the REI for the Aggregation100 is the smallest. In addition, we observe that we have the steepest slope for the Aggregation100 


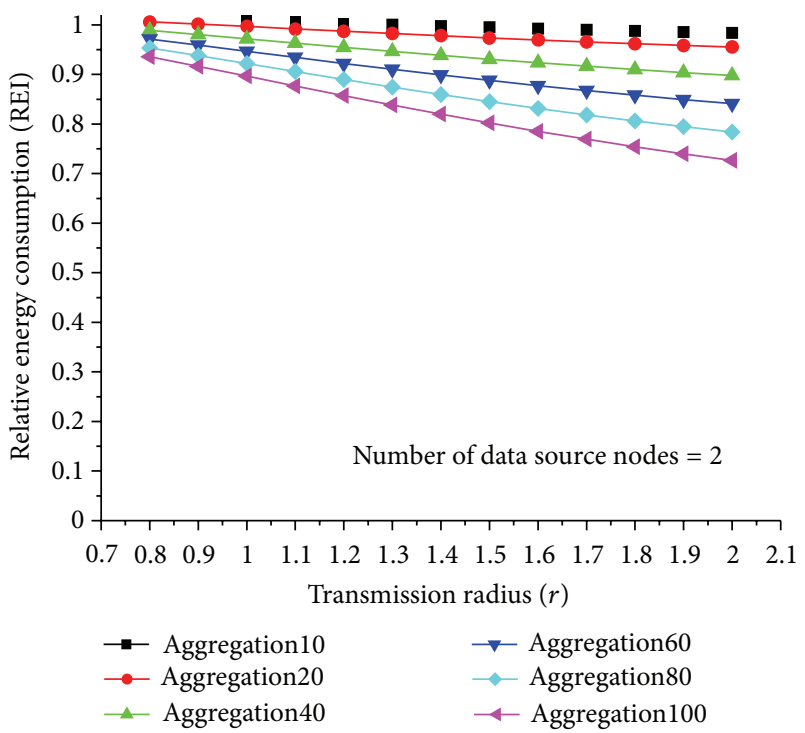

FIGURE 11: REI comparison with respect to transmission radius.

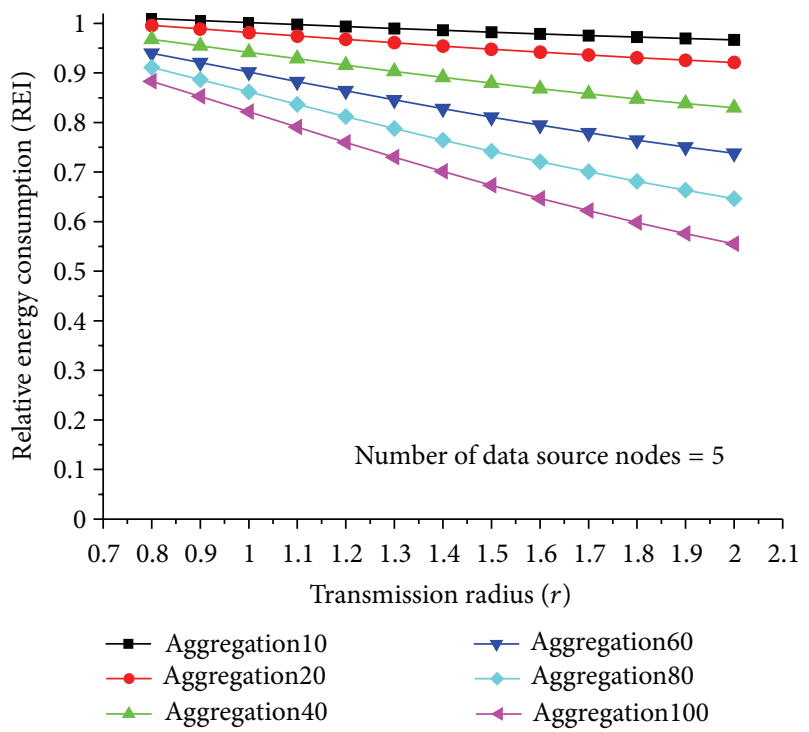

FIGURE 12: REI comparison with respect to transmission radius.

with respect to the increasing transmission radius. In other words, in large transmission radius, the higher percentage of aggregation the better energy saving there would be.

(3) REI becomes smaller as the number of aggregated nodes increases. As the number of aggregated nodes increases, the transmission energy saving from reducing the duplicated image increases. Consider the Aggregation100 scheme at $2 r$ transmission radius; we have the 0.72 REI, 0.55 REI, and 0.5 REI at Figures 11, 12 , and 13 , respectively.

Based on the previous three observations, they are consistent with the first set of experiments. That is, image aggregation should be encouraged to decrease the total power

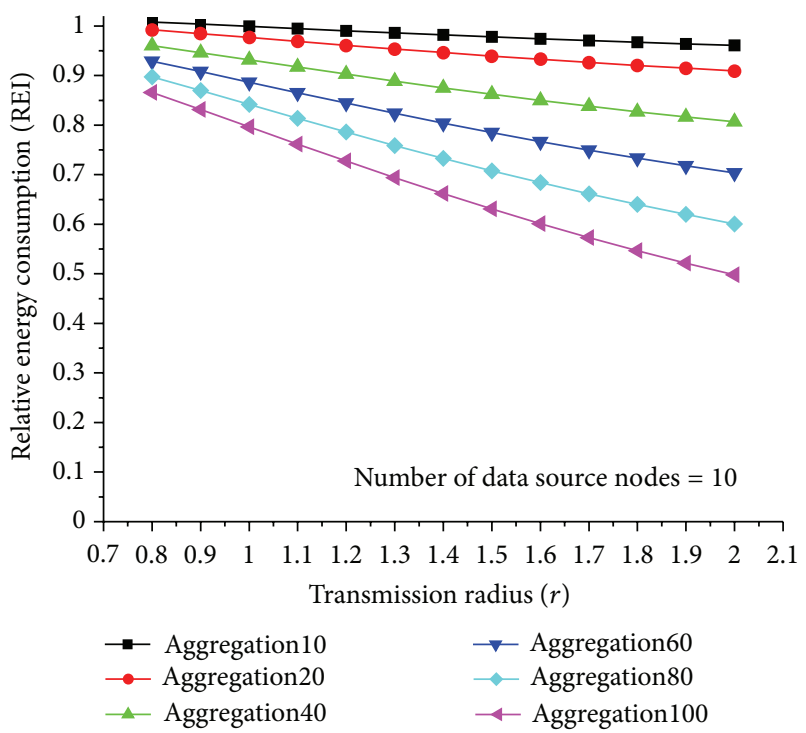

FIGURE 13: REI comparison with respect to transmission radius.

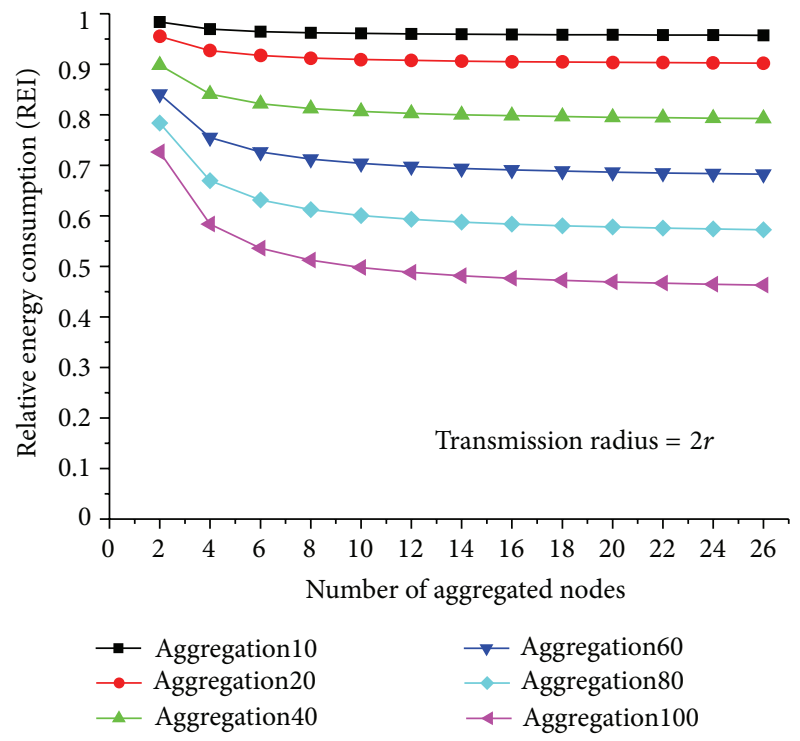

FIGURE 14: REI comparison with respect to no. of aggregated nodes.

consumption in higher degree of aggregation and large transmission radius (i.e., large $T / P$ ratio). As indicated previously, REI becomes smaller as the number of aggregated nodes increases. As a matter of fact, the REI will not always keep decreasing as the number of aggregated keeps increasing. In other words, the REI will be saturated to a minimum value. One important question to be asked is what is the value of saturated REI? The other important question is what is the number of aggregated nodes corresponding to the saturated REI? In Figure 14 and Table 4, we will answer the above two questions when the transmission radius is fixed at $2 r$.

In Table 4 , when the REI value does not reduce by $0.1 \%$ for the next number of aggregated nodes, we say the REI value is saturated and the corresponding number of aggregated nodes is denoted as the threshold for the number of aggregated nodes. 
TABLE 4: Summary of the saturated REI value and the threshold for the number of aggregated nodes.

\begin{tabular}{lcc}
\hline & Saturated REI value & $\begin{array}{c}\text { Threshold for no. of } \\
\text { aggregated nodes }\end{array}$ \\
\hline Aggregation 10 & 0.96 & 12 \\
Aggregation 20 & 0.9 & 12 \\
Aggregation 40 & 0.79 & 16 \\
Aggregation 60 & 0.68 & 18 \\
Aggregation 80 & 0.57 & 20 \\
Aggregation 100 & 0.46 & 22 \\
\hline
\end{tabular}

In other words, when REI value is saturated, increasing the number aggregated nodes does not help much in reducing the energy consumption (i.e., at most $0.1 \%$ ). These results could help determining the number of aggregated nodes under different degree of aggregation to achieve minimum energy consumption.

In this paper, until now, we only study the energy consumption on one-hop image aggregation but without addressing the multihop image aggregation strategies (i.e., network level). However, the computational results will give us some ideas on energy efficient multihop image strategies. In the WVSN, when the event occurs, the FoV of the sensors that can cover the event will become the data source nodes and send the images back to the sink. It could be expected that the number of aggregated nodes (or images) will become smaller along the path back to the sink. Hence, for the relay node on the routing path to the sink, the closer it is to the sink, the smaller number of images it will receive from downstream aggregated nodes. In addition, it could also be expected that the percent of the redundant image portion from the received images will be decreased along the routing path back to the sink. In other words, for the relay node on the routing path to the sink, the closer it is to the sink, the number of received images and the percent of the redundant image portion from the received images will be smaller. Based on the computational results that the image aggregation should be encouraged at higher percent of redundant image portion from the received images and higher number of aggregated nodes, we can conclude that the image aggregation should not be encouraged when the relay node is close to the sink. On the other hand, the image aggregation should be encouraged when the relay node is close to the data source nodes. Hence, even though the multihop routing issue is not considered in this paper, the results in this paper provide a good guideline in designing energy efficient image routing scheme in WVSN.

\section{Conclusions}

Equipping the camera on the sensor node, the capability to capture and transmit the image data opens a new application on environment monitoring and security surveillance for WVSN. However, transmitting image in WVSN is a challenging issue in power-limited WVSN network. By aggregating the images from multiple data source nodes, the identical portion of the images could be combined to save the image transmission power. This image processing needs additional node energy consumption that jeopardizes the power saving advantage from image transmission. In addition, aggregating the image from multiple data source nodes to the same relay nodes incurs MAC layer retransmission power loss. Hence, energy efficient image aggregation scheme needs to address the interplay between these three factors. In this paper, we first propose the mathematical model to capture the energy consumption from image transmission, image processing, and MAC retransmission. Then the numerical results based on the mathematical model and real WVSN sensor node (Meerkats node) are conducted to determine the best image aggregation strategy. From the numerical results in mathematical model and real WVSN sensor node, they all show that image aggregation should be encouraged to decrease the total power consumption in higher degree of aggregation and large transmission radius (i.e., large $T / P$ ratio). Increasing the number of data source nodes will also increase the multiplexing gains from aggregating multiple images to the single relay node. This indicates that the image aggregation could help to decrease the total power consumption in large number of data source nodes. However, as more data source nodes aggregated on a single node, the MAC retransmission power loss also increases. From the numerical results, it is shown that the number of data source nodes should not exceed seven to get the best tradeoff between multiplexing gain and the MAC retransmission power loss. These results not only determine the best image aggregation strategy to trade off between image transmission, image processing, and MAC retransmission but also help the routing decision in WVSN to minimize the total power consumption.

\section{Acknowledgment}

This work was supported by the National Science Council, Taiwan, under Grants no.s NSC 100-2221-E-128-002 and NSC 101-2410-H-128-005-MY2.

\section{References}

[1] S. Soro and W. Heinzelman, "A survey of visual sensor networks," Advances in Multimedia, vol. 2009, Article ID 640386, 21 pages, 2009.

[2] H. H. Yen, "Optimization-based channel constrained data aggregation routing algorithms in multi-radio wireless sensor networks," Sensors, vol. 9, no. 6, pp. 4766-4788, 2009.

[3] Y. Bai, Feature-based image comparison and its application in wireless visual sensor networks [Ph.D. dissertation], University of Tennessee, 2011.

[4] D. G. Lowe, "Distinctive image feature from scale-invariant keypoints," International Journal of Computer Vision, vol. 60, no. 2, pp. 91-110, 2004.

[5] H. Bay, A. Ess, T. Tuytelaars, and L. Van Gool, "Speeded-Up Robust Features (SURF)," Computer Vision and Image Understanding, vol. 110, no. 3, pp. 346-359, 2008.

[6] H. S. M. Coxeter, Introduction to Geometry, John Wiley \& Sons, New York, NY, USA, 2nd edition, 1969. 
[7] F. Y. S. Lin, H. H. Yen, and S. P. Lin, "A novel energyefficient MAC aware data aggregation routing in wireless sensor networks," Sensors, vol. 9, no. 3, pp. 1518-1533, 2009.

[8] H. H. Yen, F. Y. S. Lin, and S. P. Lin, "Energy-efficient datacentric routing in wireless sensor Networks," IEICE Transactions on Communications, vol. 88, pp. 4470-4480, 2005.

[9] A. Skordas, C. Chirstopoulos, and T. Ebrahimi, "The JPEG 2000 still image compression standard," IEEE Signal Processing Magazine, vol. 18, no. 5, pp. 36-58, 2001.

[10] A. Mammeri, A. Khoumsi, D. Ziou, and B. Hadjou, "Modeling and adapting JPEG to the energy requirements of VSN," in Proceedings of the 17th International Conference on Computer Communications and Networks (ICCCN '08), pp. 806-811, August 2008.

[11] R. Wagner, R. Nowak, and R. Baraniuk, "Distributed image compression for sensor networks using correspondence analysis and super resolution," in Proceedings of the IEEE International Conference on Image Processing (ICIP '03), vol. 1, pp. 597600, September 2003.

[12] B. Girod, A. Aaron, S. Raneand, and D. Rebollo-Monedero, "Distributed source coding," Proceedings of the IEEE, vol. 93, no. 1, pp. 71-83, 2005.

[13] Z. Xiong, A. D. Liveris, and S. Cheng, "Distributed source coding for sensor networks," IEEE Signal Processing Magazine, vol. 21, no. 5, pp. 80-94, 2004.

[14] K. Y. Chow, K. S. Lui, and E. Y. Lam, "Efficient on-demand image transmission in visual sensor networks," Eurasip Journal on Advances in Signal Processing, vol. 2007, Article ID 95076, 11 pages, 2007.

[15] L. Ferrigno, S. Marano, V. Paciello, and A. Pietrosanto, "Balancing computational and transmission power consumption in wireless image sensor networks," in Proceedings of the IEEE International Conference onVirtual Environments, HumanComputer Interfaces, and Measurement Systems (VECIMS '05), pp. 61-66, June 2005.

[16] C. B. Margi, R. Manduchi, and K. Obraczka, "Energy consumption tradeoffs in visual sensor networks," in Proceedings of 24th Brazilian Symposium on Computer Networks, June 2006.

[17] K. Khursheed, M. Imran, M. O’Nils, and N. Lawal, "Exploration of local and central processing for a wireless camera based sensor node," in Proceedings of the International Conference on Signals and Electronic Systems (ICSES '10), pp. 147-150, September 2010.

[18] K. W. Fan, S. Liu, and P. Sinha, "On the potential of structurefree data aggregation in sensor networks," in Proceedings of the 25th IEEE International Conference on Computer Communications (INFOCOM '06), pp. 1-12, Barcelona, Spain, April 2006.

[19] S. T. Sheu, Y. Tsai, and J. Chen, "MR² RP: the multi-rate and multi-range routing protocol for IEEE 802.11 ad hoc wireless networks," Wireless Networks, vol. 9, no. 2, pp. 165-177, 2003. 

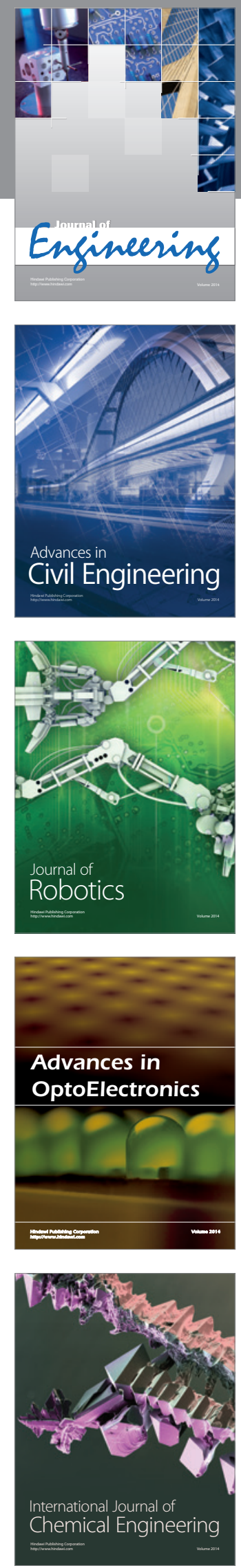

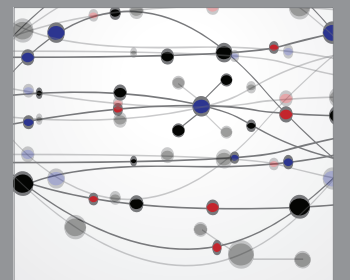

The Scientific World Journal
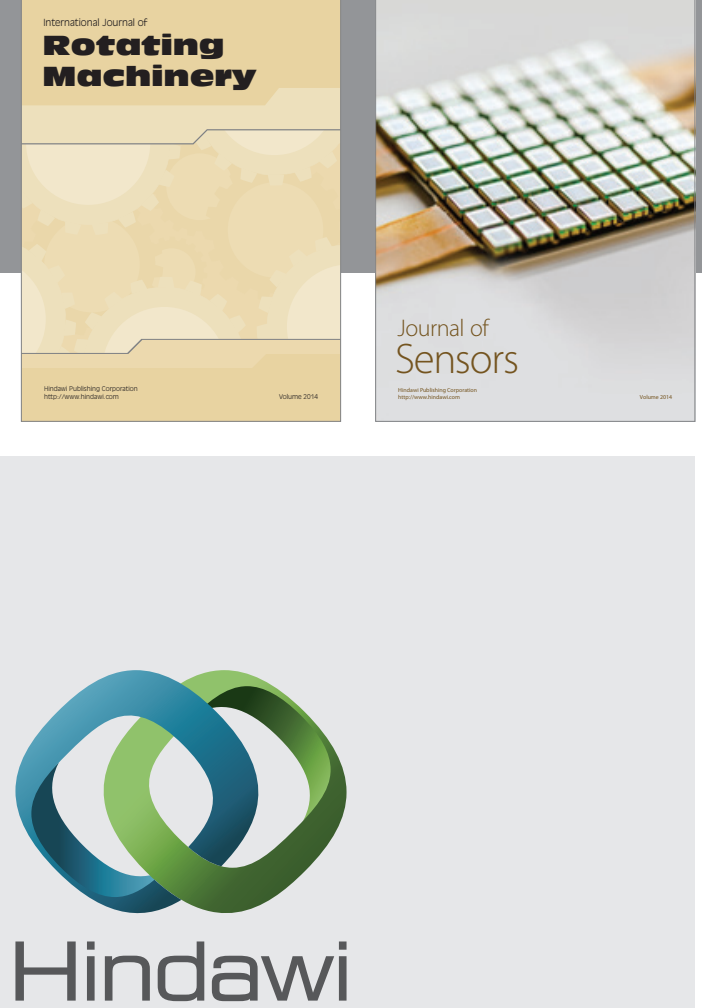

Submit your manuscripts at http://www.hindawi.com
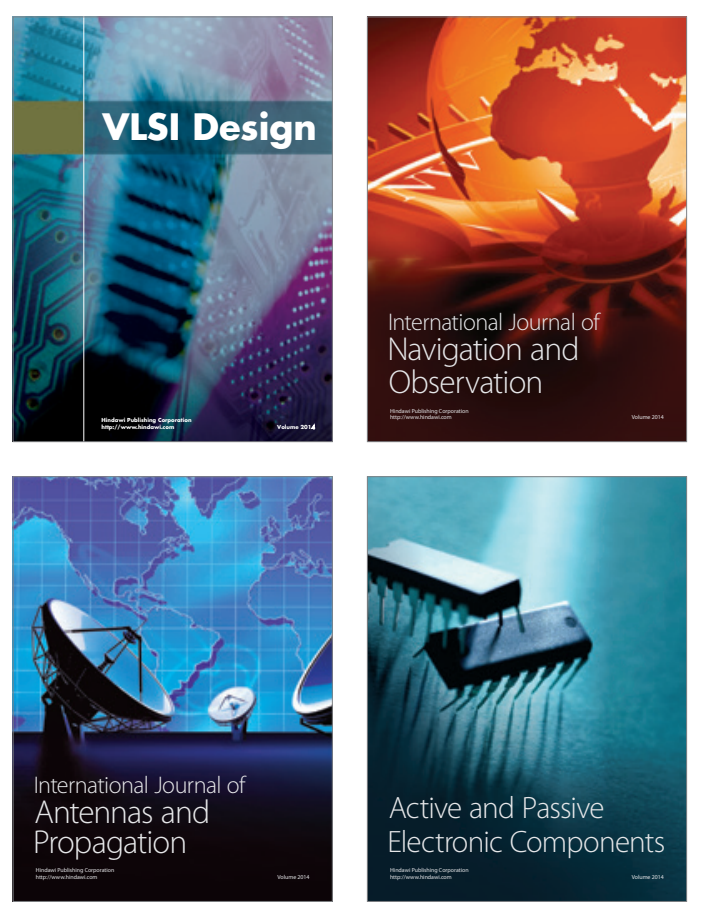
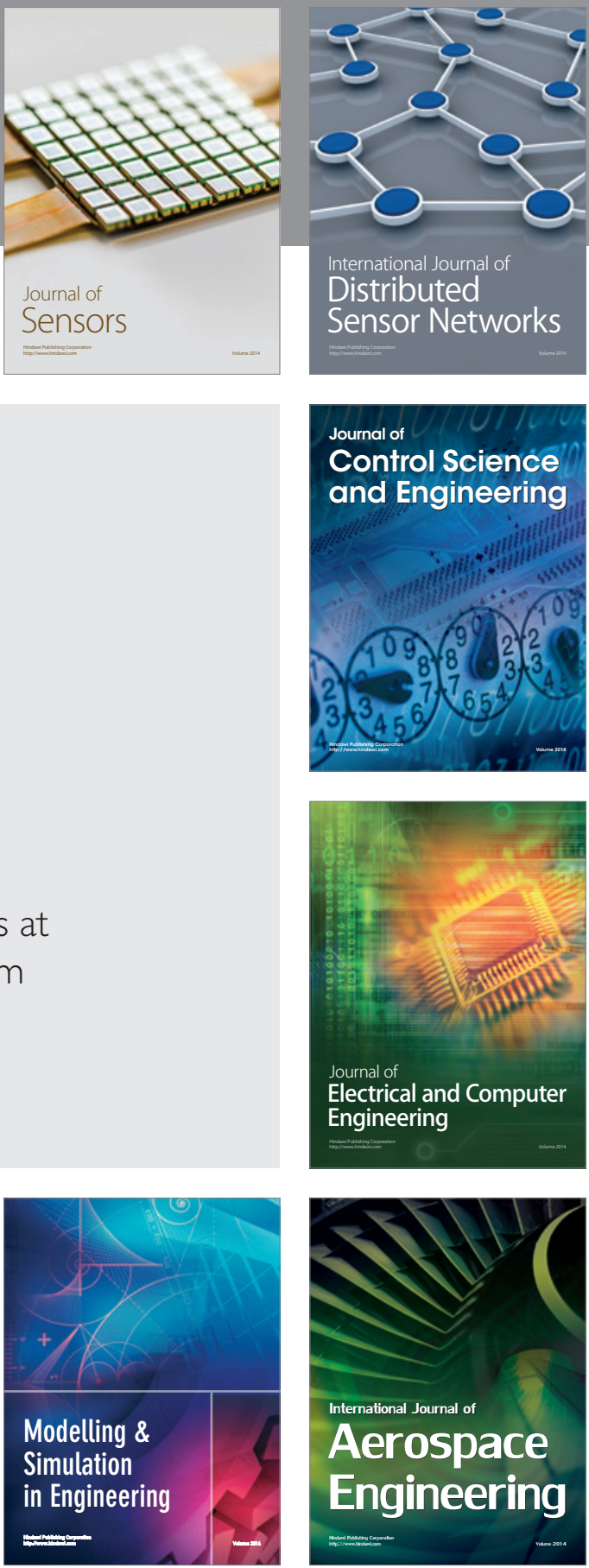

Journal of

Control Science

and Engineering
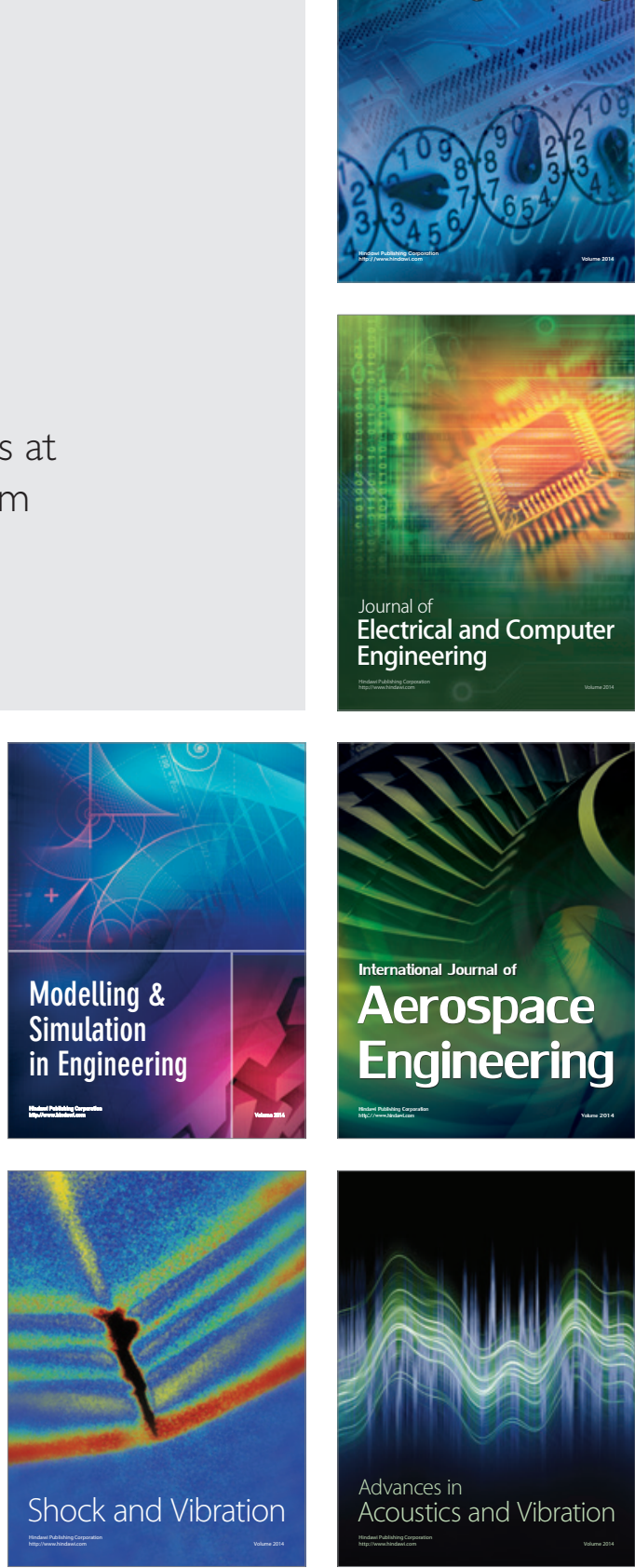